\title{
PREMEDICATION PROTOCOLS IN DENTAL PRACTICE IN NON-ALLERGIC PATIENTS
}

\author{
Angelina Kisselova ${ }^{1}$, Adriana Krasteva ${ }^{2}$, Assya Krasteva ${ }^{1}$ \\ 1) Department of Imaging and Oral Diagnostic, Faculty of Dental Medicine, \\ Medical University, 1 G. Sofiyski Blvd., Sofia 1000, Bulgaria \\ Medical Center "Detsko zdrave”, 27-29 Ami Bue Str., Sofia 1000, Bulgaria \\ 2) Department of Pharmacology and Toxicology, Faculty of Pharmacy, Medical \\ University, 2, Dunav str. Sofia 1000, Bulgaria
}

\begin{abstract}
The aim is to present some of the most prescribed premedication schemes prior to an upcoming dental analgesia in non-allergic patients. These schemes we would like to be proved as "standard protocols" in dental practice.

Key words: Premedication protocols, non-allergic patients, dental analgesia
\end{abstract}

Premedication protocols prior dental procedures (usually including and under dental anaesthesia) are usually discussed $(1,3,5)$. When choosing a drug for premedication it is of a great importance to know the benefits, the contraindications and the possible side effects that must be complied with the general condition of the patient $(4,5,6)$. Some of the most commonly prescribed schemes we proposed and presented in two categories: non-allergic patients negative to skin testing to local anesthetics and non-allergic patients positive to skin testing.

Table 1. Premedication protocols in patients with negative skin testing for hypersensitivity to local anaesthetics

\begin{tabular}{|l|l|l|}
\hline Drugs & & Premedication scheme \\
\hline H1 blockers: & & \\
Cetirizine hydrochloride (Zyrtec) & $10 \mathrm{mg} /$ daily & 3 days prior and 3 days after dental procedure \\
Levocetirizine hydrochloride (Xyzal) & $5 \mathrm{mg}$ daily & \\
Loratadine (Claritine, Roletra) & $10 \mathrm{mg}$ daily & \\
Desloratadine (Aerius) & $5 \mathrm{mg} \mathrm{daily}$ & \\
Fexofenadine (Telfast, Ewofex) & $120 \mathrm{mg} / 180 \mathrm{mg}$ daily & \\
\hline H2 blockers: & & \\
Ranitidine hydrochloride (Ranitidine, Zantac) & $300 \mathrm{mg}$ daily & 3 days prior and 3 days after dental procedure \\
Famotidine (Quamatel, Famotidine) & $40 \mathrm{mg}$ daily & \\
\hline Vitamins: & & \\
Ascorbic acid (Vitamin C) & $1000 \mathrm{mg}$ daily & 3 days prior and 3 days after dental procedure \\
\hline
\end{tabular}

*The suggested protocols are adapted for children older than 6 years.

Table 2. Premedication protocols in patients with positive skin testing for hypersensitivity to local anaesthetics

\begin{tabular}{|l|l|l|}
\hline Drugs & & Premedication scheme \\
\hline H1 blockers: & & \\
Cetirizine hydrochloride (Zyrtec) & $10 \mathrm{mg} /$ daily & 5 days prior and 5 days after dental procedure \\
Levocetirizine hydrochloride (Xyzal) & $5 \mathrm{mg}$ daily & \\
Loratadine (Claritine, Roletra) & $10 \mathrm{mg}$ daily & \\
Desloratadine (Aerius ) & $5 \mathrm{mg}$ daily & \\
Fexofenadine (Telfast, Ewofex) & $120 \mathrm{mg} / 180 \mathrm{mg}$ daily & \\
\hline
\end{tabular}




\begin{tabular}{|l|l|l|}
\hline $\begin{array}{l}\text { H2 blockers: } \\
\text { Ranitidine hydrochloride (Ranitidine, Zantac) } \\
\text { Famotidine (Quamatel, Famotidine) }\end{array}$ & $\begin{array}{l}300 \mathrm{mg} \text { daily } \\
40 \mathrm{mg} \text { daily }\end{array}$ & 5 days prior and 5 days after dental procedure \\
\hline $\begin{array}{l}\text { Vitamins: } \\
\text { Ascorbic acid (Vitamin C) }\end{array}$ & $1000 \mathrm{mg}$ daily & 5 days prior and 5 days after dental procedure \\
\hline $\begin{array}{l}\text { Corticosteroids: } \\
\text { Prednisolone tabl. }\end{array}$ & $4 \times 5 \mathrm{mg}$ daily & 5 days prior and 5 days after dental procedure \\
\hline
\end{tabular}

*The suggested protocols are adopted for children older than 6 years.

\section{CONCLISION}

It should be noted that the recommended pre-medication protocols are tentative and the approach should be always individual. The pre-medication schemes should be administrated after consultation and skin testing performed by an allergist.

\section{REFERENCES:}

1. Djerasi, E., B. Petrunov. Alergologichni problem v stomatologijata. - publisher Medizina i Fiskultura, Sofia, 1990, 245. (in Bulgarian)

2. Kisselova-Yaneva, A. Lokalni uslojnenija i inzidenti pri prilagane na lokalni anestetizi. Godishen sbornik na IMAB, VIII, 1, 2002, 76-77. (in Bulgarian)
3. Kisselova-Yaneva, A. Stomatologichna alergologia i ognistna diagnostic. Monograph, publisher "Gutenberg", Sofia, 2001, 327. (in Bulgarian)

4. Mileva, J. Klinichna alergologia. Book, publisher "Znanie", Sofia, 2001. (in Bulgarian)

5. Petrunov B., A. Kisselova-Yaneva,
V. Dimitrov et al. Klinichna imunologija, klinichna alergologija, dentalna klinichna alergologija. Book, publisher "Arso", Sofia, 2009. (in Bulgarian)

6. Mileva, J. Savremeno lechenie na alergichnite bolesti. Book, publisher "Znanie", Sofia, 1999. (in Bulgarian)

\footnotetext{
Address for correspondence:

Angelina Kisselova

Department of Oral imaging and oral diagnostic, Faculty of Dental Medicine, Medical University - Sofia

1, Sveti Georgi Sofiiski Blvd., 1000 Sofia, Bulgaria

E-mail: prof_kisselova@yahoo.com
} 